\title{
RMP ELM SUPPRESSION IN DIII-D PLASMAS WITH ITER SIMILAR SHAPES AND COLLISIONALITIES
}

by

T.E. Evans, M.E. Fenstermacher, R.A. Moyer, T.H. Osborne, J.G. Watkins, P. Gohil, I. Joseph, M.J. Schaffer, L.R. Baylor, M. Bécoulet, J.A. Boedo, K.H. Burrell, K.H. Finken, T.C. Jernigan, M. Jakubowski, C.J. Lasnier, M. Lehnen, A.W. Leonard, J. Lonnroth, E. Nardon, V. Parail, O. Schmitz, B. Unterberg, and W.P. West 


\section{DISCLAIMER}

This report was prepared as an account of work sponsored by an agency of the United States Government. Neither the United States Government nor any agency thereof, nor any of their employees, makes any warranty, express or implied, or assumes any legal liability or responsibility for the accuracy, completeness, or usefulness of any information, apparatus, product, or process disclosed, or represents that its use would not infringe privately owned rights. Reference herein to any specific commercial product, process, or service by trade name, trademark, manufacturer, or otherwise, does not necessarily constitute or imply its endorsement, recommendation, or favoring by the United States Government or any agency thereof. The views and opinions of authors expressed herein do not necessarily state or reflect those of the United States Government or any agency thereof. 


\title{
RMP ELM SUPPRESSION IN DIII-D PLASMAS WITH ITER SIMILAR SHAPES AND COLLISIONALITIES
}

by

T.E. Evans, M.E. Fenstermacher, ${ }^{\star}$ R.A. Moyer, ${ }^{\dagger}$ T.H. Osborne, J.G. Watkins, ${ }^{\ddagger}$ P. Gohil, I. Joseph, ${ }^{\dagger}$ M.J. Schaffer, L.R. Baylor, ${ }^{\natural}$ M. Bécoulet, ${ }^{\S}$ J.A. Boedo, ${ }^{\dagger}$ K.H. Burrell, K.H. Finken," T.C. Jernigan," M. Jakubowski," C.J. Lasnier, ${ }^{*}$ M. Lehnen," A.W. Leonard, J. Lonnroth, ${ }^{,}$E. Nardon, ${ }^{\S}$ V. Parail, ${ }^{\star}$ O. Schmitz, B. Unterberg," and W.P. West

This is a preprint of a paper to be submitted for publication in Nucl. Fusion.

*Lawrence Livermore National Laboratory, Livermore, California.

†University of California-San Diego, San Diego, California.

FSandia National Laboratories, Albuquerque, New Mexico.

"Oak Ridge National Laboratory, Oak Ridge, Tennessee.

${ }^{\S}$ CEA Cadarache EURATOM Association, Cadarache, France.

"Institut für Plasmaphysik, Association FZJ- EURATOM, Jülich, Germany.

${ }^{\curlyvee}$ Association EURATOM-TEKES, Helsinki University of Technology HUT, Finland.

'EURATOM/UKAEA Fusion Association, Culham Science Centre, Abingdon, UK.

\author{
Work supported by \\ the U.S. Department of Energy \\ under DE-FC02-04ER54698, W-7405-ENG-48, DE-FG02-04ER54758, \\ DE-FG02-05ER54809, DE-AC04-94AL85000, and DE-AC05-000R22725
}

\section{GENERAL ATOMICS PROJECT 30200} MAY 2007 


\begin{abstract}
Large Type-I Edge Localized Modes (ELMs) are completely eliminated with small $n=3$ resonant magnetic perturbations (RMP) in low average triangularity, $\bar{\delta}=0.26$, plasmas and in ITER Similar Shaped (ISS) plasmas, $\bar{\delta}=0.53$, with ITER relevant collisionalities $v_{e}^{*} \leq 0.2$. Significant differences in the RMP requirements and in the properties of the ELM suppressed plasmas are found when comparing the two triangularities. In ISS plasmas, the current required to suppress ELMs is approximately $25 \%$ higher than in low average triangularity plasmas. It is also found that the width of the resonant $q_{95}$ window required for ELM suppression is smaller in ISS plasmas than in low average triangularity plasmas. An analysis of the positions and widths of resonant magnetic islands across the pedestal region, in the absence of resonant field screening or a self-consistent plasma response, indicates that differences in the shape of the $q$ profile may explain the need for higher RMP coil currents during ELM suppression in ISS plasmas. Changes in the pedestal profiles are compared for each plasma shape as well as with changes in the injected neutral beam power and the RMP amplitude. Implications of these results are discussed in terms of requirements for optimal ELM control coil designs and for establishing the physics basis needed in order to scale this approach to future burning plasma devices such as ITER.
\end{abstract}




\section{INTRODUCTION}

Type-I Edge Localized Modes (ELMs) [1] are a significant concern in magnetically confined toroidal fusion plasmas because they can rapidly erode plasma facing material surfaces, reduce the coupling efficiency of rf antennas, cause noise in feedback circuits used to control core MHD modes and interfere with diagnostic measurements of critical operating parameters. ELMs have been suppressed in the DIII-D tokamak [2] over a wide range of pedestal collisionalities $v_{e}^{*}=q_{95} R \varepsilon^{-3 / 2} \lambda_{e}^{-1}$ [3] and plasma shapes with resonant magnetic perturbations (RMPs) that have toroidal mode numbers $n=3$. Here, $R$ is the major radius, $\varepsilon(\equiv a / R)$ is the inverse aspect ratio, $a$ is the minor radius and $\lambda_{e}\left(=\mathrm{v}_{T e} \tau_{e}\right.$, the product of electron thermal velocity $\mathrm{v}_{T e}$ and collision time $\tau_{e}$ ) is the mean free path for electron collisions. In high average triangularity $\bar{\delta}=\left(\delta_{\text {upper }}+\delta_{\text {lower }}\right) / 2=0.53$ plasmas with moderate collisionalities $v_{e}^{*} \approx 1$, Type-I ELMs are replaced by small intermittent events with a coherent amplitude modulation of $\sim 130 \mathrm{~Hz}$ [4,5]. In these discharges, an $n=3$ RMP pulse with a vacuum field peak spectral amplitude approximately equal to that of the DIII-D field-errors in the edge plasma is used to create a dense set of magnetic islands across the pedestal. These islands are produced by an RMP from the DIII-D I-coil [6] and have poloidal mode numbers ranging from $m=9$ to $m=14$ across the pedestal when the safety factor on the $95 \%$ normalized flux surface $q_{95}$ is between 3.5 and 3.9. Outside this $q_{95}$ range there is little or no effect on the ELMs indicating a resonant condition on the size and distribution of the islands across the pedestal. When this $q_{95}$ resonant condition is satisfied, ELMs are suppressed by the applied RMP without any appreciable change in the pedestal pressure profile [7] suggesting that the RMP directly couples to the eigenmode structure of the ELMs, which then changes the stability of the modes.

In low collisionality plasmas with $v_{e}^{*} \leq 0.2$ and $\bar{\delta}=0.26$, ELMs are completely eliminated over a $q_{95}$ resonant window ranging from 3.55 to 3.85 . The minimum $m, n=11,3$ RMP spectral amplitude required for ELM suppression under these conditions lies between $\delta b_{r}^{(11,3)} B_{T}^{-1}=1.7 \times 10^{-4}$ and $\delta b_{r}^{(11,3)} B_{T}^{-1}=2.6 \times 10^{-4}$ for I-coil currents of 2 and $3 \mathrm{kA}$ respectively [8]. Here, $B_{T}$ is the toroidal magnetic field on axis. This RMP field amplitude is approximately 8-9 times larger than that required in the $\bar{\delta}=0.53 v_{e}^{*} \approx 1$ case and does not include effects due to the response of the plasmas to the field such as flow or pressure screening $[9,10]$. In these low collisionality plasmas, ELMs are suppressed during a reduction in the pedestal pressure profile. The pedestal pressure is reduced by an apparent change in the global particle balance which reduces the density without appreciably affecting the energy transport and is controlled by the amplitude of the I-coil current [11]. This change in the pressure profile moves the pedestal into a stable 
peeling-ballooning mode operating region [12] suggesting that ELM suppression occurs in this case without the RMP directly interacting with the ELM eigenmode.

In this paper we compare RMP ELM suppression results in low collisionality plasmas $v_{e}^{*} \leq 0.2$ with average triangularities of $\bar{\delta}=0.26$ and $\bar{\delta}=0.53$. We refer to these as the low triangularity and ITER Similar Shaped (ISS) plasmas respectively. ISS plasmas are dimensionally similar to an ITER Scenario 2 plasma [13] but have been scaled down to fit in the DIII-D vacuum vessel. Here, we focus on similarities and differences in these plasma shapes that provide insight into the physics of ELM suppression, referred to as RMP H-modes, at ITER relevant collisionalities and results in ISS plasmas that can be used to help predict the behavior of RMP H-modes in future devices such as ITER. 


\section{EXPERIMENTAL BACKGROUND AND OBSERVATIONS}

\section{A. Plasma shape and general operating parameters}

Between the 2005 and 2006 operating period the DIII-D lower cryopump baffle was extended, as shown in Fig. 1, to facilitate pumping in plasmas with higher triangularities similar to those expected in ITER. This new pumping configuration makes it possible to carry out RMP H-mode experiments with $v_{e}^{*} \leq 0.2$ in ISS plasmas having $\delta_{\text {upper }}=0.36$, $\delta_{\text {lower }}=0.70$ and $\bar{\delta}=0.53$.

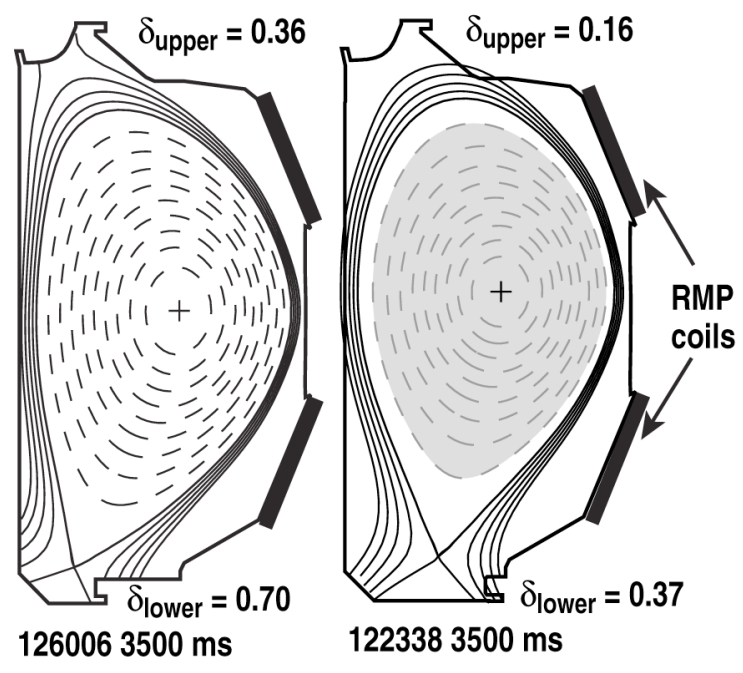

Fig. 1. Plasma shapes used to carry out $v_{e}^{*} \leq 0.2$ RMP H-mode experiments (right) prior to February 2006 with $\bar{\delta}=0.26$ and (left) beginning in February 2006 with $\bar{\delta}=0.53$. Note that the gray flux surfaces in the right hand figure are used as color key for data associated with this shape in several of the following figures.

Here, we describe results from RMP H-modes with $v_{e, n e o}^{*}=0.2 \rightarrow 0.04$ where both the $n=3$ DIII-D I-coil and the DIII-D correction coil (C-coil), operated in $n=1$ fielderror feedback mode [14], are used simultaneously. These discharges are configured for strong lower single null divertor pumping, as shown in Fig. 1, with a plasma currents $I_{p}$ ranging between 1.0 and 1.6 MA. The toroidal magnetic field on axis $B_{T}$ ranges between 1.9 and $2.1 \mathrm{~T}$ while normalized beta $\beta_{N}=\beta /\left(I_{p} / a B_{T}\right)$ is varied from $\beta_{N}=1.4$ to $\beta_{N}=2.2$. In general, these plasmas are typical H-mode discharges with ITER 98(y2) scaling factors of approximately 1.0 and elongations of $\sim 1.8$. In order to maintain good density control needed to achieve the lowest possible collisionality, the outer strikepoint of these lower single null diverted plasmas is positioned near the cryopump baffle as illustrated in Fig. 1. 


\section{B. Characteristics of RMP ELM suppression in low collisionality plasmas}

As shown in Figs. 2(a) and 2(b), ELMs are completely eliminated in ISS plasmas with $\bar{\delta}=0.53$ and in low triangularity plasmas with $\bar{\delta}=0.26$. In ISS plasmas slightly larger I-coil currents and RMP fields are needed to completely eliminate ELMs. Figure 2(c) shows the minimum I-coil current needed to completely eliminate ELMs in an ISS plasma (126006 with an I-coil current of $4 \mathrm{kA}$ corresponding to $\left.\delta b_{r}^{(11,3)} B_{T}^{-1}=3.4 \times 10^{-4}\right)$ and that needed to eliminate ELMs in a low triangularity plasma (122338 with and I-coil current of $3 \mathrm{kA}$ corresponding to $\delta b_{r}^{(11,3)} B_{T}^{-1}=2.8 \times 10^{-4}$ ). As discussed below, this requirement on RMP spectral amplitude is consistent with a difference in the pedestal $q$ profile for the two shapes although when ELMs are completely eliminated $q_{95}$ lies within a specific resonant window in both cases.

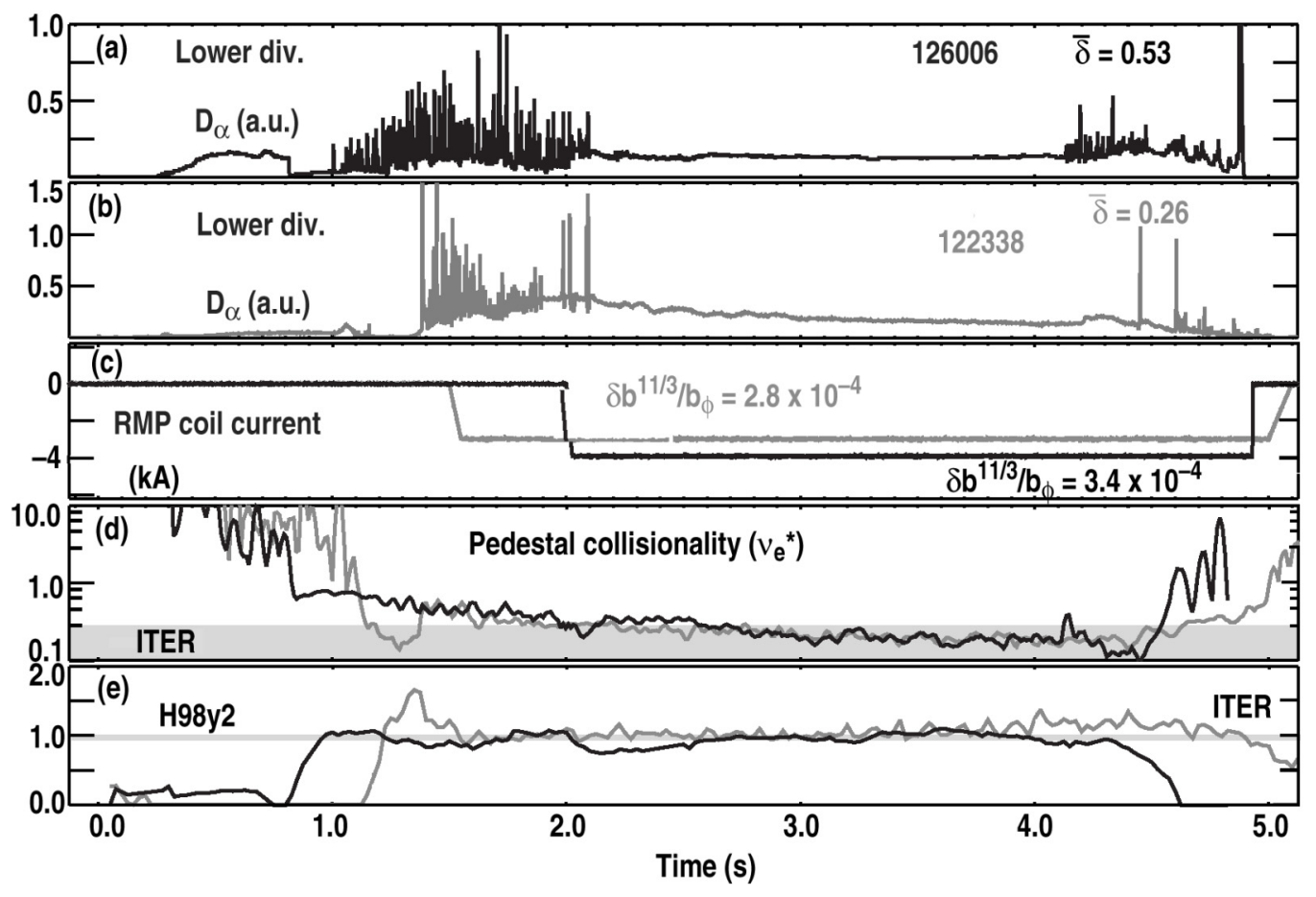

Fig. 2. (a) Lower divertor $D_{\alpha}$ response to the RMP in an ISS plasma with $\bar{\delta}=0.53$, (b) lower divertor $D_{\alpha}$ response to the RMP in a low triangularity plasma with $\bar{\delta}=0.26$, (c) timing and amplitude of the I-coil pulse in the ISS plasmas (black) and the low triangularity plasma (gray), (d) evolution of the electron pedestal collisionality and (e) H-factor for a low triangularity (gray) and an ISS plasma (black).

In discharge 122338 the I-coil pulse begins while $q_{95}$ is outside the resonant window ( $q_{95} \sim 4$ at $t=1.5 \mathrm{~s}$ ) causing an increase in frequency and a reduction in amplitude of the ELMs. This behavior is typical when RMP fields are applied outside the resonant window [15]. Once $q_{95}$ drops into the resonant window, $q_{95}=3.7$ at $t=2.0 \mathrm{~s}$ in 
discharge 122338, ELMs are completely eliminated. It is also noted, that in discharge 126006 a neutral beam that applies a torque opposite to the direction of the toroidal rotation (counter-NBI torque) is turned on at $4.0 \mathrm{~s}$. This is done intentionally to acquire data on the effects of rotational screening as the toroidal rotation is reduced during the counter-NBI phase of the discharge. In this particular discharge, the counter-NBI reduces the toroidal rotation and triggers small ELM-like burst on the divertor $D_{\alpha}$ signals. As discussed below, this behavior is correlated with the onset of a core MHD mode when the toroidal rotation drops below a specific level.

\section{RMP effects on pedestal profiles}

Changes in the pedestal profiles during RMP H-modes in low triangularity plasmas with $\bar{\delta}=0.26$ compared to similar discharges with ELMs are discussed in Refs. 11 and 15. It is interesting to note that there are significant differences in the pedestal profiles during RMP H-modes with $\bar{\delta}=0.26$ as compared to ISS $(\bar{\delta}=0.53)$ RMP H-mode plasmas. Here, $B_{T}$ was held fixed at $1.92 \mathrm{~T}$ while $q_{95}=3.63\left(I_{p}=1.41 \mathrm{MA}\right)$ in the $\bar{\delta}=0.26$ plasma and $q_{95}=3.54\left(I_{p}=1.54 \mathrm{MA}\right)$ in the ISS plasma. Figure 3 shows how an RMP pulse affects the pedestal profiles in a typical ISS discharge at $4 \mathrm{kA}$ (125916) with full ELM suppression compared to a low triangularity discharge (122336) with an I-coil current of $3 \mathrm{kA}$. In the ISS plasma the injected neutral beam power $\left(P_{i n j}\right)$ is 7.2 MW at this time in the discharge $(t=2.35 \mathrm{~s})$ and the pedestal density is significantly higher than in the $\bar{\delta}=0.26$ plasma with $P_{i n j}=9.4 \mathrm{MW}$. Although $P_{i n j}$ is somewhat lower in the ISS plasma this has a relatively small impact on the pedestal profiles as discussed below. In the ISS plasma both the electron and ion pedestal temperatures are lower than in the $\bar{\delta}=0.26$ case. The net result is that the pedestal pressure profile is significantly wider in the ISS case as seen in Fig. 3(d) and the absolute value of the pressure gradient has a smaller peak value that is shifted inward away from the separatrix and significantly broader as shown in Fig. 3(e). There is also more shear in the toroidal rotation across the pedestal in the ELM suppressed ISS plasma as seen in Fig. 3(f). A broadening of the pedestal pressure profile is characteristic of both ELMing and ELM suppressed ISS plasmas compared to $\bar{\delta}=0.26$ plasmas and is believed to be related to a reduction in the stable peeling-ballooning operating region in ISS plasmas [12]. In ISS discharge $125916 P_{i n j}$ was increased from 7.2 MW to $9.4 \mathrm{MW}$ at $t \sim 2.55 \mathrm{~s}$, as shown in Fig. 4(b), to assess the impact of changes in the input power on pedestal profiles and ELM stability. Figure 4(a) shows the behavior of a typical lower divertor $D_{\alpha}$ signal during the RMP pulse in this discharge. Here, we see that when the RMP is turned on at $t=2.0 \mathrm{~s}$ there is a sharp step up in the $D_{\alpha}$ baseline and it remains somewhat constant during the rest of the RMP pulse. In low triangularity RMP H-modes there is usually a transient increase in the divertor $D_{\alpha}$ signals followed by a steady drop over the next several seconds as seen in Fig. 2(b). For the case shown in Fig. 4, we also note that the ELM frequency increases initially when the RMP turns on and that after several cycles 
ELMs are completely eliminated. After the increase in $P_{i n j}$ there appears to be a small upward drift in the $D_{\alpha}$ baseline and the ELMs do not reappear.
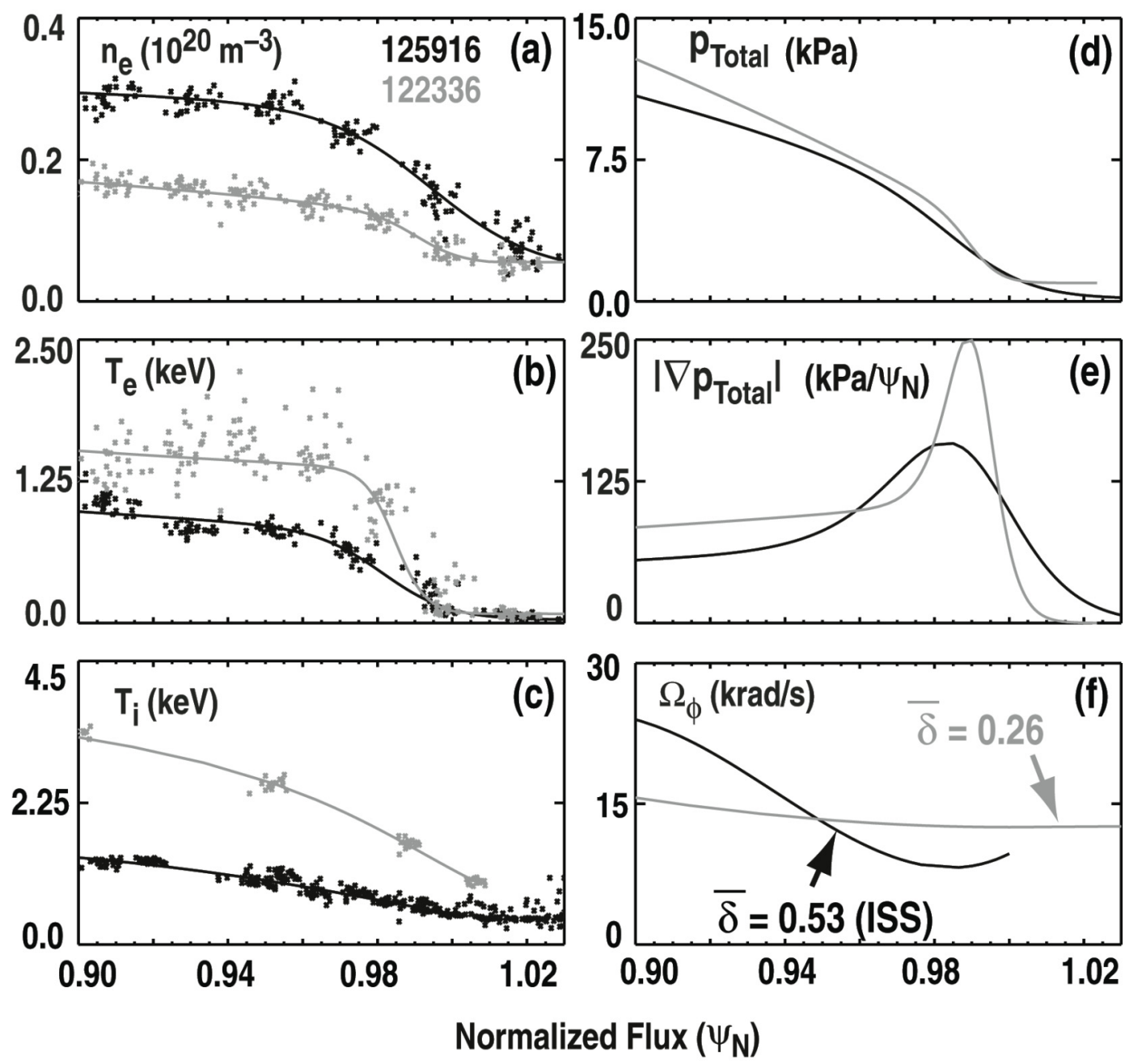

Fig. 3. (a) Pedestal density, (b) electron temperature, (c) ion temperature, (d) total pressure, (e) absolute value of the total pressure gradient and (f) toroidal rotation during a 4 kA RMP pulse in an ISS plasma with $\bar{\delta}=0.53$ (discharge 125916 black) and during a $3 \mathrm{kA}$ RMP pulse in a low triangularity plasma with $\bar{\delta}=0.26$ (discharge 122336 gray).

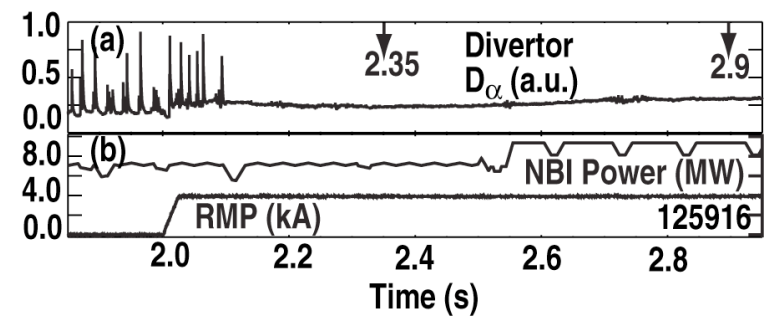

Fig. 4. (a) $D_{\alpha}$ response to a 4 kA RMP pulse and (b) NBI power in an ISS plasma with $\bar{\delta}=0.53$ (discharge 125916). Note the increase in NBI power from 7.2 MW to 9.4 MW at $t \sim 2.55 \mathrm{~s}$. 
As shown in Fig. 5(a), the pedestal density profile remains constant after increasing $P_{i n j}$ in discharge 125916 while the electron temperature profile and $T_{e}{ }^{\text {ped }}$ increase. Figure 5(b) shows the $T_{e}$ profile at $2.9 \mathrm{~s}$ (black) and at $2.35 \mathrm{~s}$ (gray) with $P_{i n j}=9.4 \mathrm{MW}$ and 7.2 MW respectively. When $P_{i n j}$ is increased both the height and width of the high pressure gradient region $\left|\nabla p_{\text {total }}\right|$ increases, as seen in Fig. 5(c), without destabilizing ELMs. Thus, the ELM suppressed state is robust to changes in $P_{i n j}$ and pedestal pressure over the range tested.

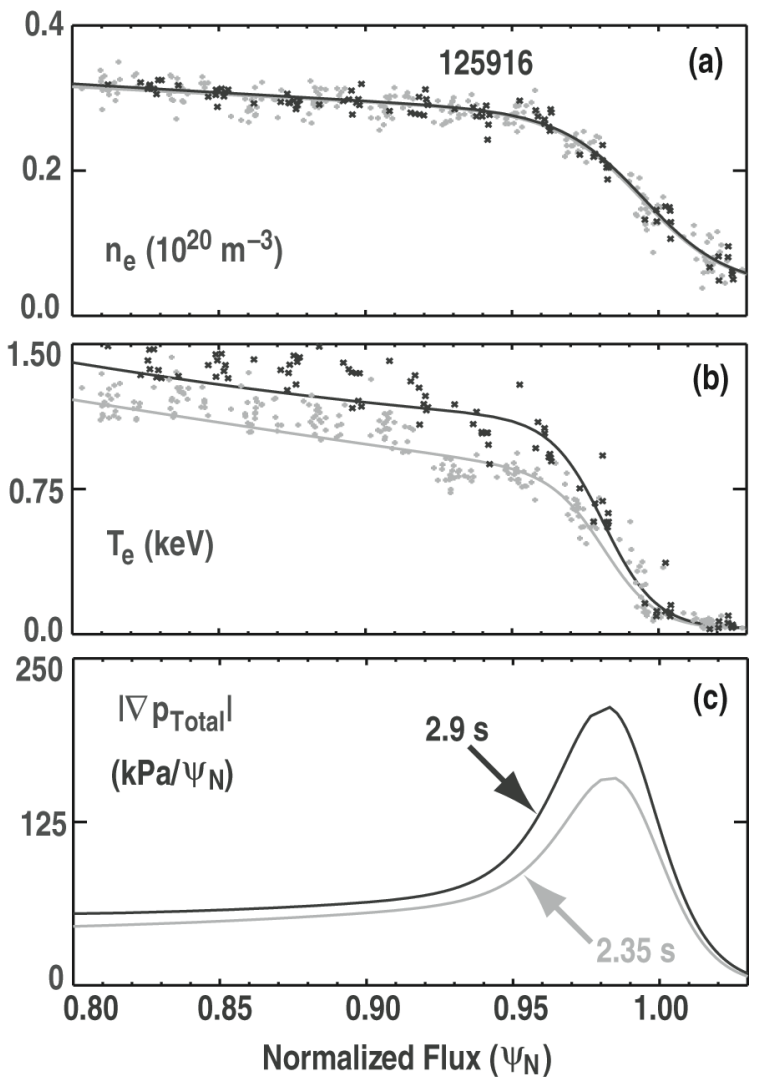

Fig. 5. (a) Pedestal density, (b) electron temperature, and (c) absolute value of the total pressure gradient during a $4 \mathrm{kA}$ RMP pulse in an ISS plasma with $\bar{\delta}=0.53$ (discharge 125916) with an injected neutral beam power of $7.2 \mathrm{MW}$ at $2.35 \mathrm{~s}$ (gray) and with 9.4 MW at $2.9 \mathrm{~s}$ (black) as shown in Fig. 4.

Understanding the effect of the RMP fields on the pedestal profiles is essential for comparing ELM suppression with stability theory. Figure 6(a)-(c) provides a comparison of the ELM behavior seen on a lower divertor $D_{\alpha}$ signal near the outer strikepoint with various I-coil currents in ISS plasmas. As the I-coil current is increased there is a strong monotonic reduction in the pedestal density Fig. $6(\mathrm{~d})$ and the $C^{6+}$ toroidal rotation increases with each step in the I-coil current Fig. 6(h). The change in ion temperature is most pronounced when going from 0 to $4 \mathrm{kA}$, particularly in the high gradient region, and drops slightly at the top of the pedestal when going from 4 to $6.3 \mathrm{kA}$. The electron 
temperature profile, Fig. 6(f), shows a steady reduction in pedestal width and height with increasing I-coil current. The net effect on the total pressure profile is seen in Fig. $6(\mathrm{~g})$ where the absolute value of the pressure gradient $\left|\nabla p_{\text {total }}\right|$ is plotted. Here, we see that full ELM suppression occurs when the pressure gradient profile resides somewhere between the red $4 \mathrm{kA}$ curve and the black $6.3 \mathrm{kA}$ curve suggesting that the critical region required for ELM stabilization lies between $\psi_{N} \sim 0.80-0.97$. Looking at the various components of $\left|\nabla p_{\text {total }}\right|$ in Figs. 6(d)-(f) for the red to black profiles shows that a significant fraction of the change in the region from $0.80-0.95$ is due to a flattening of the density profile with relatively small contributions from changes in the ion and electron temperature.
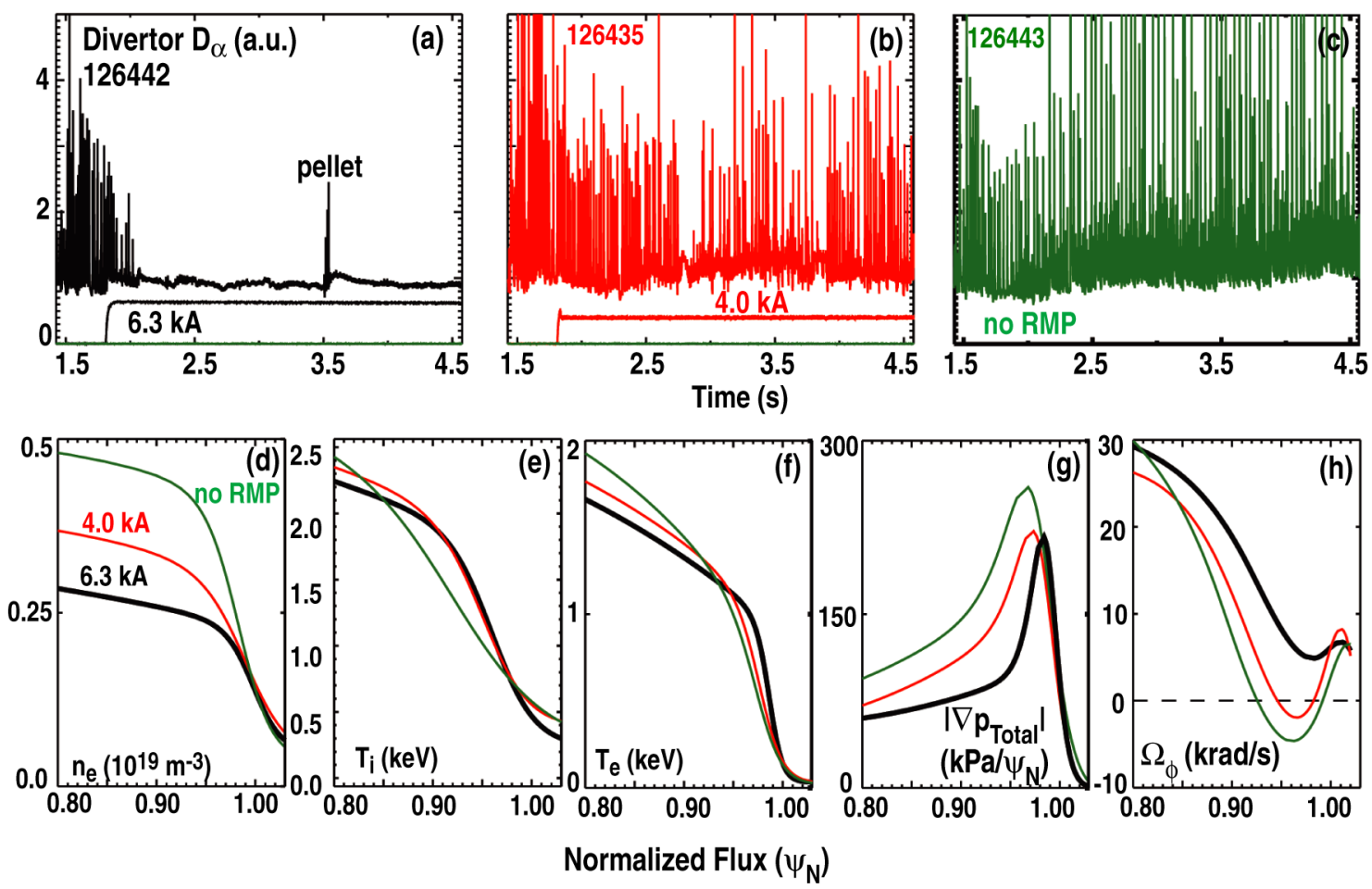

Fig. 6. Lower divertor $D_{\alpha}$ signals characterizing the ELM characteristics in similar ISS plasmas with $n=3$ I-coil currents of (a) $6.3 \mathrm{kA}$, (b) $4.0 \mathrm{kA}$, and (c) $0 \mathrm{kA}$. Pedestal profiles showing the (d) density, (e) ion temperature, (f) electron temperature, (g) absolute value of the total pressure gradient, and (h) $C^{6+}$ toroidal rotation for the 3 I-coil currents shown in (a), (b) and (c) where black, red and green correspond to $6.3 \mathrm{kA}, 4.0 \mathrm{kA}$ and $0 \mathrm{kA}$ respectively.

We also note that the $6.3 \mathrm{kA}$ ELM suppressed discharge (126442) had a single $1.8 \mathrm{~mm}$ fueling pellet injected at $3.5 \mathrm{~s}$ from the high field side of the discharge. This pellet triggered 3 small $D_{\alpha}$ bursts in the lower divertor as shown in Fig. 7(b). The first significant $D_{\alpha}$ burst is delayed by about $17 \mathrm{~ms}$ after the pellet enters the plasma although there is an initial indication of a perturbation in the $D_{\alpha}$ signal at 3.505 s. Figure 7(a) shows the characteristics of the lower divertor $D_{\alpha}$ signals during the ELMs before the 
I-coil pulse in this discharge. The black trace views a region near the inner strikepoint and the red trace a region near the outer strikepoint. The $D_{\alpha}$ bursts following the deuterium fueling pellet are smaller than those seen during ELMs. The line integrated density increase due to the fueling pellet decays with an e-folding time of $\sim 0.25 \mathrm{~s}$ increasing the $D_{\alpha}$ baseline somewhat as the particles from the pellet flow into the lower divertor [Figs. 6(a) and 7(b)].

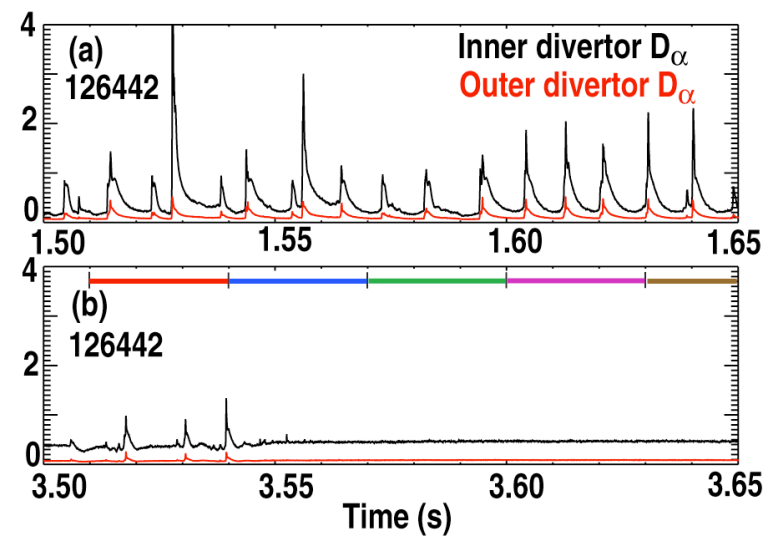

Fig. 7. Lower divertor $D_{\alpha}$ (a.u.) signals near the inner strikepoint (black) and the outer strikepoint (red) showing (a) Type-I ELMs before the I-coil pulse and (b) 3 small bursts following the injection of a deuterium fuelling pellet injected from the high field side of the discharge at $3.5 \mathrm{~s}$. The multi-colored bands along the top of (b) indicate the averaging times used for the pedestal profile reconstructions shown in Fig. 8.

It should be noted that we do not know if these bursts are associated with a rapid drop in the pedestal energy, as is the case with Type-I ELMs, or if they are related to intermittent convective transport events that do not tend to carry much energy compared to a Type-I ELM [16]. Nevertheless, the evolution of the pedestal profiles following the injection of the fueling pellet in this discharge yields some interesting information about the nature of these $3 D_{\alpha}$ bursts. Figure 8(a) shows a pedestal reference density profile during the RMP ELM suppressed phase prior to the injection of the fueling pellet at $t=3400 \mathrm{~ms}$ (the black curve) and a series of five profiles averaged over $30 \mathrm{~ms}$ following the pellet (the multicolor bars at the top of Fig. 7(b) indicate the averaging intervals). We see that the pedestal density increases significantly during the interval when the three $D_{\alpha}$ bursts are observed (red) while the electron temperature and $C^{6+}$ rotation drop Figs. 8(b) and $8(\mathrm{~d})$ respectively. During this time, the peak in $\left|\nabla \mathrm{p}_{\text {total }}\right|$ increases and the profile narrows (as shown by the red curve) compared to the reference $\left|\nabla p_{\text {total }}\right|$ profile (as shown by the black curve). Interestingly, the peak in $\left|\nabla p_{\text {total }}\right|$ increases by about a factor of 1.3-2 during the four time intervals following the initial $3 D_{\alpha}$ bursts but the plasmas remain quiescent during this entire period. This data again suggests that it is not the height of the peak in $\left|\nabla p_{\text {total }}\right|$ that determines the stability of Type-I ELMs. In addition, as shown in Fig. $8(\mathrm{~d})$, the $C^{6+}$ rotation initially drops following the pellet and recovers as the pedestal profiles evolve. 

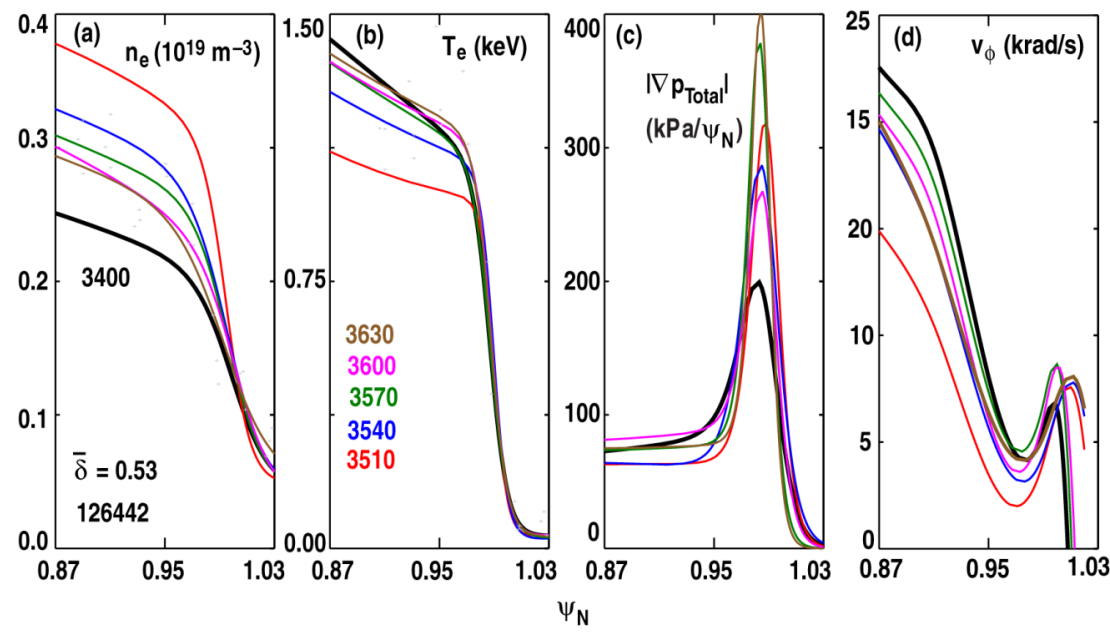

Fig. 8. The evolution of the pedestal (a) density, (b) electron temperature, (c) absolute value of total pressure gradient and (d) $C^{6+}$ toroidal rotation during the I-coil pulse averaged over $30 \mathrm{~ms}$ for 5 intervals following the injection of a $1.8 \mathrm{~mm}$ deuterium fueling pellet (see color coded bars in Fig. 7(b) for the timing of the intervals). The black profiles at $t=3400 \mathrm{~ms}$ provide references prior to the injection of the pellet.

\section{Safety factor effects on RMP ELM suppression}

One consistent feature seen in all RMP H-modes, either with $\bar{\delta}=0.53$ in high or low collisionality plasmas or with $\bar{\delta}=0.26$ at low collisionality, is a distinct range of $q_{95}$ values (a resonant window) over which ELMs are suppressed. The width and position of the window is somewhat different for each shape and collisionality as mentioned above. In ISS plasmas the width of the ELM suppression window is $\Delta_{q 95}^{E L M}=3.62-3.52=0.10$ as seen in Figs. 9(b) and 9(c), with an I-coil current of 4 kA [Fig. 9(a)]. In a similar $\bar{\delta}=0.26$ plasma the ELM suppression window is typically $\Delta_{q 95}^{E L M}=3.85-3.55=0.30$ [11] with an I-coil current of $3 \mathrm{kA}$ but is somewhat more variable with plasma density and $P_{i n j}$ than in ISS plasmas.

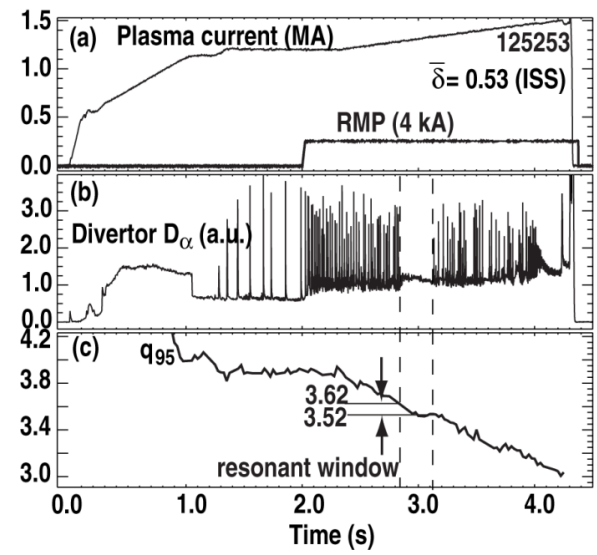

Fig. 9. (a) Plasma and I-coil current, (b) lower divertor $D_{\alpha}$ signal showing ELM suppression window, and (c) $q_{95}$ resonant window during a $4 \mathrm{kA}$ RMP pulse in an ISS plasma with $\bar{\delta}=0.53$ (discharge 125253). 


\section{E. Toroidal rotation effects on RMP ELM suppression}

As mentioned above, when a counter-NBI torque is applied to a co-rotating ISS plasma, bursts in the $D_{\alpha}$ signals, which look similar to those seen during Type-I ELMs but smaller and higher in frequency, are sometimes observed [as shown in Fig. 2(a) starting at $4.0 \mathrm{~s}]$. This behavior is correlated to the amount of counter-NBI torque applied and the evolution of the $C^{6+}$ toroidal rotation. Figure 10 shows a comparison of two ISS RMP H-mode discharges in which a counter-NBI torque was applied starting at $4.0 \mathrm{~s}$
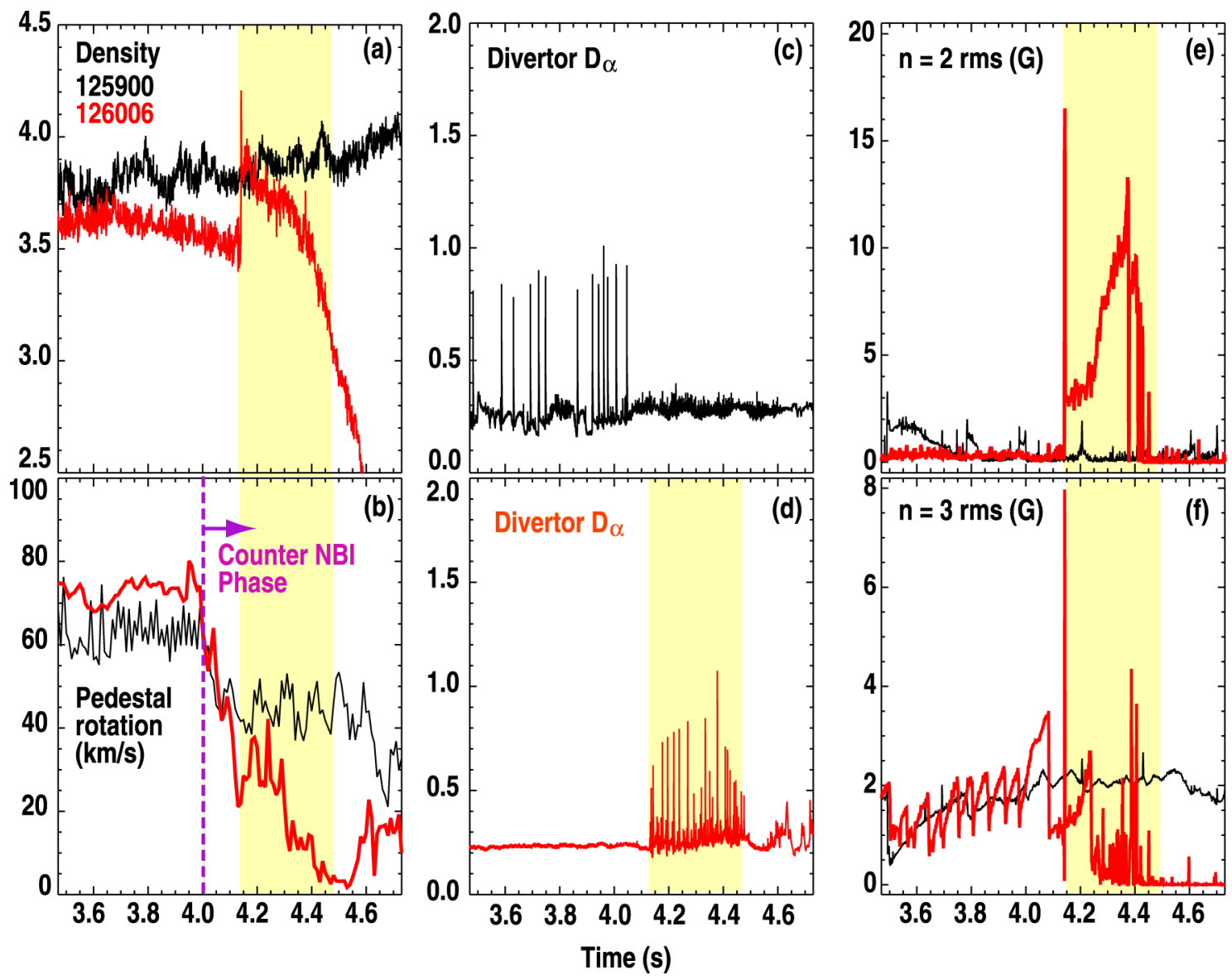

Fig. 10. Comparison of the plasma (a) density, (b) $C^{6+}$ toroidal rotation, (c) and (d) lower divertor $D_{\alpha}$ near the outer strikepoint (e) rms level of $n=2$ MHD activity and (f) rms level of $n=3$ MHD activity in two similar discharges (125900-black and 126006-red) with different levels of counter-NBI torque applied at $t=4.0 \mathrm{~s}$. The shaded region indicates the time over which the core MHD and edge $D_{\alpha}$ bursts appear to be correlated in 126006 .

[see Fig. 10(b)]. Here, the RMP is on at a constant level over the entire time interval shown in the figure. In one case, the applied RMP level is just below that required for full ELM suppression (discharge 125900 shown in black). Here, ELMs are present until the counter-NBI torque is applied as shown in Fig. 10(c) and are fully suppressed following the drop in toroidal rotation. In this discharge the applied counter-NBI torque is smaller 
than that in discharge 126006 (red) and the toroidal rotation drops from about $60 \mathrm{~km} / \mathrm{s}$ prior to the counter-NBI down to about $45 \mathrm{~km} / \mathrm{s}$ during the counter-NBI [Fig. 10(b)]. We note that the MHD signals shown in Figs. 10(e) and 10(f) remain relatively constant across this transition.

In discharge 126006 with more counter-NBI torque the rotation drops rapidly from about $70 \mathrm{~km} / \mathrm{s}$ at $4.0 \mathrm{~s}$ to $20 \mathrm{~km} / \mathrm{s}$ at $4.2 \mathrm{~s}$ as indicated by the red curve in Fig. 10(b). At this point, a large core MHD mode is triggered (the red trace in Fig. 10(e)) and small, high frequency $D_{\alpha}$ bursts appear as shown by the red trace in Fig. 10(d). Ultimately, the core MHD mode locks which is followed by an $H \rightarrow L$ transition. We note a reduction in the amplitude of the $D_{\alpha}$ bursts while the core mode is slowing down and locking. This suggests a strong correlation between the core mode and the $D_{\alpha}$ bursts, which is not typically seen during Type-I ELMs in these discharges.

\section{F. Differences in divertor and pedestal impurities with changes in triangularity}

In ISS plasmas the divertor recycling is relatively high while impurity emission from the divertors and the core plasma is rather low. During RMP H-modes the divertor recycling increases slightly and the impurity emission remains low. This is in contrast to the behavior seen in $\bar{\delta}=0.26 \mathrm{RMP} H$-mode plasmas [17] where the divertor goes into a sheath limited regime during the ELM suppressed phase with the RMP. Signatures of the sheath limited condition in the $\bar{\delta}=0.26$ cases included increased carbon impurity emission throughout the divertor legs, localized high emission points at the gaps between tiles on the divertor targets, and very negative floating potential at the targets. None of these signatures are seen in ELM suppressed ISS discharges.

A direct comparison of similar discharges in the two shapes shows that the response of the boundary plasma to RMP ELM control is qualitatively different. The time evolution of parameters for comparable low $v^{*}$ plasmas in the $\bar{\delta}=0.26$ (black) and in the $\bar{\delta}=0.53$ ISS configuration (red) is shown in Fig. 11. The ELMs are suppressed in both discharges, although a few transient events persist in the $\bar{\delta}=0.53$ ISS plasma. The pedestal density $n_{e}^{p e d}$, normalized beta $\beta_{N}=\beta /\left(I_{p} / a B_{T}\right)$, H-mode confinement factor H98y 2 and $q_{95}$ are comparable, although $n_{e}^{\text {ped }}$ is slightly higher in the ISS discharge and the level of core MHD activity (g) is lower, especially in the early part of the RMP phase. Images of divertor $C^{2+}$ visible emission (CIII, $\lambda=465 \mathrm{~nm}$ ) from the pre-RMP ELMing phase in an ISS discharge and the ELM controlled phase (Fig. 12) show the emission remains near the divertor targets at both the inner and outer strikepoints (ISP and OSP) in both phases. There is clear evidence of splitting of the ISP emission into multiple filaments during the ELM controlled phase with the RMP. The spacing of these filaments varies with plasma conditions in some discharges. However, the large increase in CIII emission along the divertor legs up to the X-point seen in the $\bar{\delta}=0.26$ experiments was not seen in the ISS plasmas. Also, the very local emission at the gaps between divertor 


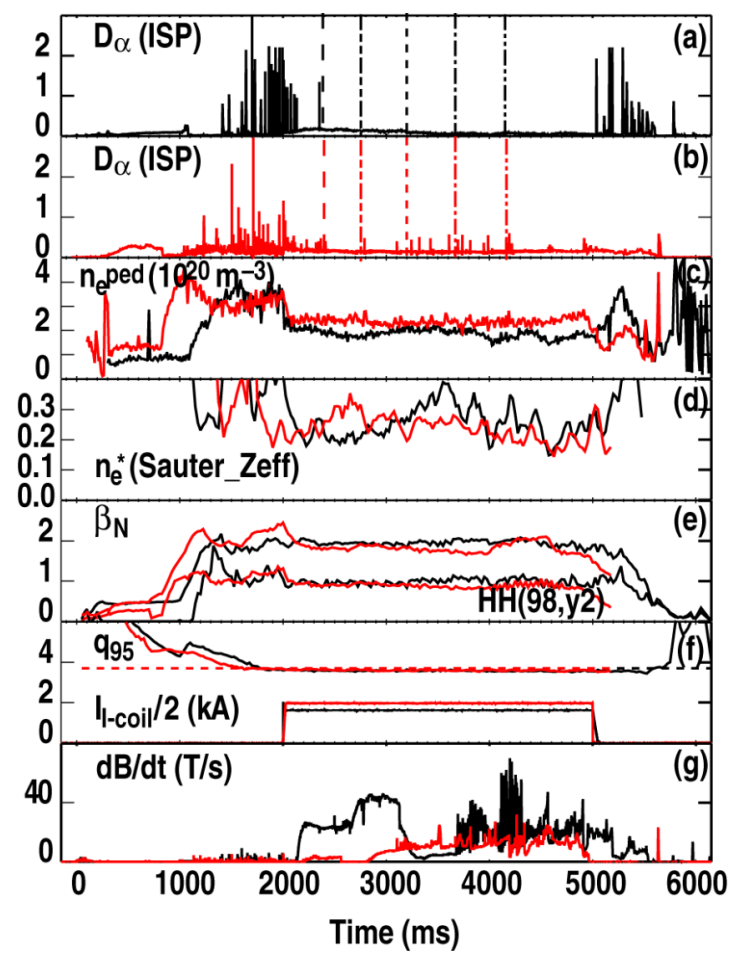

Fig. 11. Comparison of the RMP ELM-suppressed discharge evolution at low $v_{\mathrm{e}}^{*}$, showing ISP $D_{\alpha}$ (a.u.) for (a) the low $\delta$ shape (black) vs (b) the high $\delta$ ISS configuration (red). Evolution of (c) pedestal density, $n_{\mathrm{e}}^{\text {ped }}\left(10^{20} \mathrm{~m}^{-3}\right)$, (d) pedestal electron collisionality [18] $v_{\mathrm{e}}^{*}$ corrected for $Z_{\text {eff }}(\mathrm{e})$ normalized beta, $\beta_{N}=\beta /\left(I_{p} / a B_{T}\right)$ and energy confinement enhancement factor (H98y2) [13,19], (f) safety factor at 95\% flux $\left(q_{95}\right)$ and I-coil current $I_{\text {Icoil }}(\mathrm{kA})$ and $(\mathrm{g}) d B / d t(\mathrm{~T} / \mathrm{s})$ for both discharges.
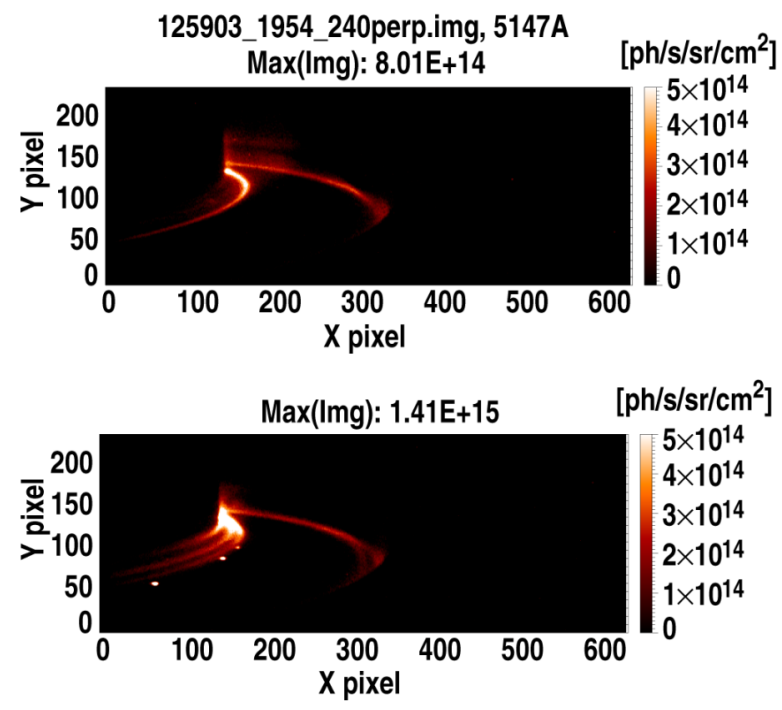

Fig.12. Tangential view of divertor CIII (465 nm) emission (a) in pre-RMP ELMy H-mode $(t=1.95 \mathrm{~s})$ and (b) during RMP ELM suppression $(t=3.79 \mathrm{~s})$ in an ISS plasma (discharge 125903). Tokamak centerpost on the left - strong emission seen near inner and outer strikepoint regions. 
target tiles is substantially less in the ISS plasmas. In addition, the radial profiles of $Z_{\text {eff }}$ in the core plasma for various times before and during the RMP ELM suppression in the two discharges (Fig. 13) show that the build up of carbon impurity seen in $\bar{\delta}=0.26$ plasmas (black) is not seen in the ISS configuration (red). $Z_{\text {eff }}$ reaches about 2.2 in the pedestal (2.0 on axis) during the ELM suppressed phase with $\bar{\delta}=0.53$, compared to $Z_{\text {eff }}=3.3-3.4$ in the edge (3.0 on axis) with $\bar{\delta}=0.26$. Finally, the floating potential on the divertor targets during the ELM controlled phase was much less negative in the ISS plasmas (-90 V compared with $-150 \mathrm{~V})$.

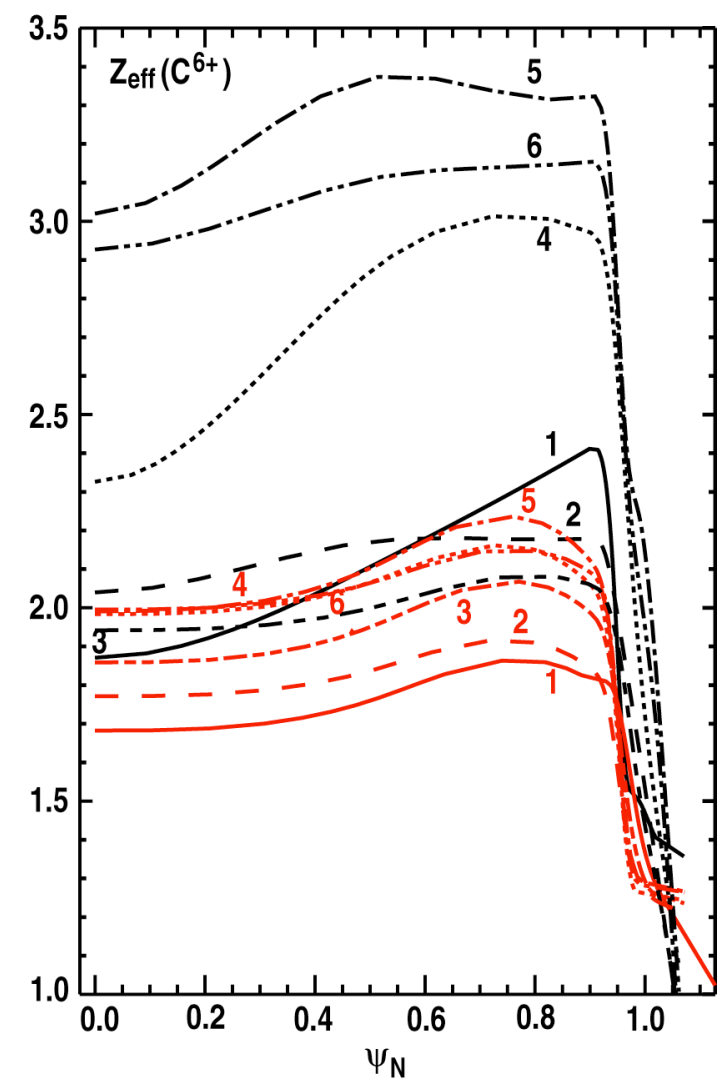

Fig.13. Profiles of $Z_{\text {eff }}$ due to fully ionized $C^{6+}$ for the low $\delta$ baseline discharge (black) vs the high $\delta$ ISS discharge (red) from Fig. 11. Profiles are shown at the times indicated by vertical lines in Fig. 11 (using the same line styles).

The signatures described above strongly suggest that the divertor in ISS RMP $\mathrm{H}$-modes remain in a high recycling condition during the ELM suppression. This positive result is likely due to a combination of factors that are different from the $\bar{\delta}=0.26$ experiments. The $\bar{\delta}=0.53$ ISS geometry favors detachment of the ISP and traps neutrals in the divertor better (shorter ionization m.f.p) than the $\bar{\delta}=0.26$ shape with both strikepoints on a flat divertor target. Also, the gaps between divertor target tiles were substantially reduced when the new $\bar{\delta}=0.53$ pumping baffle was installed in DIII-D between the two experiments, which tends to reduce tile edge effects and the propensity 
for enhanced carbon sputtering from those regions. Finally, ELM suppression in the $\bar{\delta}=0.53$ ISS RMP H-modes is obtained at slightly higher density than for the $\bar{\delta}=0.26$ experiments. All of these factors are consistent with qualities required of an RMP ELM suppressed H-mode scenario in ITER and future devices. 


\section{DISCUSSION}

Based on the data given above, we see that there are significant differences in the plasma response to the applied RMP fields in ITER relevant, low collisionality plasmas with high average triangularity compared to low average triangularity plasmas (cf. Fig. 1). Understanding the origin of these differences is important for developing a more comprehensive physics basis needed for coil designs in future tokamaks such as ITER. In particular, the fact that the I-coil current required for the complete elimination of ELMs has to be increased by $25 \%$ in ISS plasmas with high average triangularity compared to that required in low average triangularity plasmas has direct implications for RMP ELM suppression coil designs in ITER.

To better understand why larger I-coil currents are needed to suppress ELMs in DIII-D ISS plasmas we have calculated the widths and positions of $n=3$ magnetic islands for an ISS case (126006) and a $\bar{\delta}=0.26$ case (123301). The results are given in Fig. 14 where the I-coil current in discharge 123301 is $3.2 \mathrm{kA}$ and $4 \mathrm{kA}$ in the ISS case (126006). The island widths are not significantly different in these two cases but the magnetic shear is higher from the top of the pedestal $(\sim 0.9)$ out to the separatrix in the ISS plasma with $\bar{\delta}=0.53$ which puts the last good flux surface between the $m / n=-8 / 3$ and $-9 / 3$ islands rather than between the $m / n=-9 / 3$ and $-10 / 3$ islands in the $\bar{\delta}=0.26$ plasma.

Thus, the width of the island overlap region required for ELM suppression is the same in both cases but $25 \%$ more I-coil current is needed to achieve this width in the ISS plasma. The effect of the higher shear in the ISS plasma is to reduce the distance between the resonant surfaces while increasing the $m / n$ mode amplitude required to obtain islands of comparable width compared to the $\bar{\delta}=0.26$ plasma. This results in an overall compression of the island positions in $\psi_{N}$ and determines which islands leave gaps with good flux surfaces. For example, the center of the $m / n=-9 / 3$ island, near the top of the pedestal, moves from $\psi_{N}=0.91$ in the ISS plasma to $\psi_{N}=0.87$ in the $\bar{\delta}=0.26$ plasma and the $n=3$ radial field perturbation required for a comparable island width drops from $b_{r}=6.1 \mathrm{G}$ to $b_{r}=5.1 \mathrm{G}$.

It should be pointed out that this analysis only gives the relative change between these two configurations based on $n=3$ I-coil and field-error contributions. A more accurate estimate of the island overlap region requires the calculation of other contributions to the spectrum, such as $n=1$ and $n=2$ from the C-coil and field-errors, since these can fill in gaps between the $n=3$ islands. In addition, effects due to resonant field screening need to be included for a quantitative estimate. Finally, more accurate calculations of the 
equilibrium, including contributions due to the ion pressure and bootstrap current are needed in order to make meaningful comparisons with changes in the total pressure gradient profiles such as those shown in Fig. 6(g). Nevertheless, we see from the straightforward $n=3$ analysis of the island overlap regions above that the width of the implied stochastic boundary layer covers the entire extent of the unperturbed pressure pedestal including the region between $\psi_{N}=0.95$ and 0.90 where the largest changes in $\left|\nabla p_{\text {total }}\right|$ are observed in Fig. 6(g). Finally, we note that $q_{95}=3.52$ in the ISS discharge and 3.83 in the $\bar{\delta}=0.26$ discharge, which suggests that the change in the $q$ profile also affects the width and position of the ELM suppression window in a manner consistent with the experimental observations discussed above.

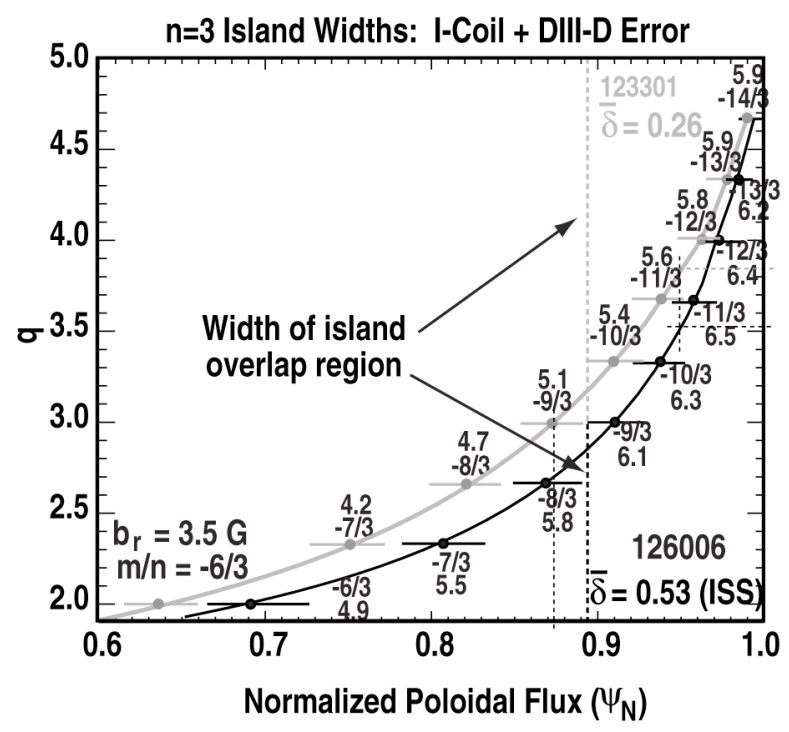

Fig. 14. $n=3$ magnetic islands widths and positions in normalized poloidal flux $\left(\psi_{N}\right)$ during a $4 \mathrm{kA}$ I-coil current in an ISS plasma with $\bar{\delta}=0.53$ (discharge 126006) and a $\bar{\delta}=0.26$ plasma (123301) with a $3.2 \mathrm{kA}$ I-coil current. $n=3$ islands are produced by the I-coil plus field-errors in both cases. The magnitude of the radial perturbation field $\left(b_{r}\right)$ along with the poloidal/toroidal mode numbers $(\mathrm{m} / \mathrm{n})$ are given for each island.

Thus, the assumption that the edge stochastic layer needs to extend beyond the top of the pressure pedestal appears to provide a useful criterion for the design of ELM control coil systems and may provide us with information that can be used to help isolate potential physics mechanisms responsible for ELM suppression. As noted above, the primary effect of the increased field with an I-coil current above $4 \mathrm{kA}$ involves a change in the density profile in the region between $\psi_{N}=0.95$ and 0.80 . This directly affects the pressure profile and $\left|\nabla p_{\text {total }}\right|$ as well as the current density profile by modifying the bootstrap contribution with changes in the pressure profile. Previously, it has been pointed out that the electrostatic potential in the stochastic region can be affected when field lines are connected to the divertor target plates and that this can lead to the 
formation of complex electric field patterns that may result in an enhancement of the convective particle transport [11].

In addition, the critical island overlap hypothesis leads to a realization that increasing the toroidal mode number of the perturbation reduces the coil current required since the number of islands between integer $q$ values, $N_{\text {islands, }}$, scales as $n-1$ although the amplitude of the perturbation falls off with increasing poloidal mode number assuming the distance between the coil and the resonant surfaces remains constant. In other words, optimizing the toroidal mode number of the perturbation can reduce the coil current required since the Chirikov parameter scales as $\sqrt{n s}$. This hypothesis also highlights the fact that field-errors, although small, can be quite important if they happen to fill in gaps in the ELM control spectrum at critical points in the pressure profile. Finally, the island overlap model implies that the width of the $q_{95}$ resonant window can be expanded by either increasing magnetic shear or the amplitude of the spectral components located in the region between $\psi_{N}=0.95$ and 0.80 . The exact transition to overlap is essentially a nonlinear process since the shape of the $q$ profile and the spectral composition of the RMP affect the positions and widths of the islands.

A quantitative analysis of the island overlap hypothesis will eventually require a detailed understanding of resonant magnetic field screening in rotating high $\beta$ plasmas. Although the data presented in Fig. 10 suggests an enhancement of the field penetration as the toroidal rotation is reduced, the enhancement appears to be relatively small. In one case, discharge 125900 (black) with an I-coil current just below that required for good ELM suppression, ELMs are completely eliminated when the toroidal rotation is reduced by approximately $25 \%$ suggesting the possibility that a gap in the island overlap profile is filled in by an increase in the island widths as more of the field penetrates into the pedestal region. The other case shown in Fig. 10, 126006 (red), also suggests an enhancement in the field penetration as the toroidal rotation is reduced. In this case ELMs are completely suppressed prior to the reduction in toroidal rotation but as the plasma spins down, large core MHD modes, perhaps destabilized by low $m$ harmonics of the field-errors or the $n=3$ I-coil perturbation, are generated and modulate in amplitude in a fashion that seems to be correlated with the $D_{\alpha}$ bursts seen in the divertor signals.

Additional experimental data is needed to quantitatively test the island overlap model and assess the effects of rotation on resonant field screening and island widths. In particular, understanding the physics involved in the density pump out, how it is related to changes in the field structure in the plasmas and why changes occur preferentially in some regions as the current in the I-coil and the toroidal rotation vary are important ingredients in evaluating the scalability of RMP ELM suppression to future devices. 


\section{SUMMARY AND CONCLUSIONS}

Large Type-I Edge Localized Modes (ELMs) are completely eliminated with small $n=3$ resonant magnetic perturbations (RMP) in low average triangularity, $\bar{\delta}=0.26$, plasmas and in ITER Similar Shaped (ISS) plasmas, $\bar{\delta}=0.53$, with ITER relevant collisionalities $v_{e}^{*} \leq 0.2$. Significant differences in the properties of the ELM suppressed plasmas are found when comparing the two triangularities. ISS plasmas have wider pressure pedestals and higher shear in the toroidal rotation than $\bar{\delta}=0.26$ plasmas during RMP H-modes. Conditions at the divertor target plates are significantly different in the two cases resulting in higher recycling and lower impurity levels during ELM suppression in ISS plasmas. It is also found, that the injection of high field side deuterium fueling pellets in ELM suppressed ISS plasmas creates a series of recycling bursts in the divertor that are smaller than those associated with Type-I ELMs and do not persist beyond $40 \mathrm{~ms}$ following the pellet. Reducing the toroidal rotation in ISS plasmas, by applying a counter-NBI torque, affects the stability of both the core and pedestal plasma during an RMP pulse. These core and edge MHD modes behave differently depending on the toroidal flow velocity near the top of the pedestal.

In ISS plasmas, the current required to suppress ELMs is approximately 25\% higher than in low average triangularity plasmas while that the width of the resonant $q_{95}$ window required for ELM suppression is smaller in ISS plasmas than in low average triangularity plasmas. An analysis of the positions and widths of resonant magnetic islands across the pedestal region, in the absence of resonant field screening or a selfconsistent plasma response, indicates that differences in the shape of the $q$ profile may explain the need for higher RMP coil during ELM suppression in ISS plasmas. Comparing the region of island overlap with change in the pressure gradient profiles required for complete ELM elimination provides a physics based constraint on the RMP mode spectrum that can be used to optimize coil designs. In addition, an accurate validation of the island overlap model including the effects of edge bootstrap currents on the $q$ profile and resonant field screening due to plasma flows will provide key information on the scalability of this approach to burning plasma devices and on the fundamental physics mechanisms involved in RMP ELM suppression. These island overlap validation experiments are in progress on DIII-D and will be discussed in a future paper. 


\section{REFERENCES}

[1] M.E. Fenstermacher, A.W. Leonard, P.B. Snyder, et al., Plasma Phys. Control. Fusion 45, 1597 (2003).

[2] J.L. Luxon, Nucl. Fusion 42, 614 (2002).

[3] A. Loarte, G. Saibene, R. Sartori, et al., Plasma Phys. Control. Fusion 45, 1549 (2003).

[4] T.E. Evans, R.A. Moyer, P.R. Thomas, et al., Phys. Rev. Lett. 92, 235003-1 (2004).

[5] R.A. Moyer, T.E. Evans, T.H. Osborne, et al., Phys. Plasmas 12, 056119 (2005).

[6] G.L. Jackson, et al., 2003 Controlled Fusion and Plasma Physics CD-ROM P-4.47 Proc. 30th EPS Conf. St. Petersburg.

[7] T.E. Evans, R.A. Moyer, J.G. Watkins, et al., Nucl. Fusion 45, 595 (2005).

[8] K.H. Burrell, T.E. Evans, E.J. Doyle, et al., Plasma Phys. Control. Fusion 47, B37 (2005).

[9] R. Fitzpatrick, Phys. Plasmas 5, 3325 (1998).

[10] A. Boozer, Phys. Plasmas 12, 0925404 (2005).

[11] T.E. Evans, K.H. Burrell, M.E. Fenstermacher, et al., Phys. Plasmas 13, 056121 (2006).

[12] T.H. Osborne, P.B. Snyder, K.H. Burrell, et al., 2007 Phys. Plasmas in press.

[13] Tokamak Physics Basis 2007 Nucl. Fusion in press.

[14] J.T. Scoville and R.J. La Haye, Nucl. Fusion 43, 250 (2003).

[15] T.E. Evans, R.A. Moyer, K.H. Burrell, et al., Nat. Phys. 2, 419 (2006).

[16] J.A. Boedo, D.L. Rudakov, E. Hollmann, et al., Phys. Plasmas 12, 072516 (2005.

[17] M.E. Fenstermacher, T.E. Evans, R.A. Moyer, et al., 2007 J. Nucl. Mater. in press.

[18] O. Sauter, et al., Phys. Plasmas 6, 2834 (1999).

[19] ITER Physics Basis Document Nucl. Fusion 39, 2137 (1999). 


\section{ACKNOWLEDGMENTS}

This work was supported by the U.S. Department of Energy under DE-FC0204ER54698，W-7405-ENG-48， DE-FG02-04ER54758， DE-FG02-05ER54809， DEAC04-94AL85000, and DE-AC05-00OR22725. 